OPEN ACCESS

Edited by:

Hao Liu,

Southern Medical University, China

Reviewed by:

Zhicheng Xue,

Sun Yat-Sen University Cancer Center,

China

Amit Gupta,

All India Institute of Medical Sciences,

Rishikesh, India

*Correspondence:

Haibo Qiu

qiuhb@sysucc.org.cn

Zhiwei Zhou

zhouzhw@sysucc.org.cn

Yingbo Chen

chenyb@sysucc.org.ch

${ }^{\dagger}$ These authors have contributed equally to this work and share first authorship

Specialty section:

This article was submitted to Gastrointestinal Cancers,

a section of the journal

Frontiers in Oncology

Received: 16 August 2020 Accepted: 21 May 2021

Published: 08 July 2021

Citation:

Zhang $M$, Ding $C, X u L$, Ou B, Feng $S$, Wang G, Wang W, Liang Y, Chen $Y$, Zhou Z and Qiu H (2021) Comparison of a Tumor-Ratio-Metastasis Staging

System and the 8th AJCC TNM Staging System for Gastric Cancer.

Front. Oncol. 11:595421.

doi: 10.3389/fonc.2021.595421

\section{Comparison of a Tumor-Ratio- Metastasis Staging System and the 8th AJCC TNM Staging System for Gastric Cancer}

\author{
Miaoquan Zhang ${ }^{1+}$, Chao Ding ${ }^{1 \dagger}$, Lin $\mathrm{Xu}^{2 \dagger}$, Biyi Ou ${ }^{1}$, Shoucheng Feng ${ }^{1}$, Guoqiang Wang ${ }^{3}$, \\ Wei Wang ${ }^{1}$, Yao Liang ${ }^{1}$, Yingbo Chen ${ }^{1 *}$, Zhiwei Zhou ${ }^{1 *}$ and Haibo Qiu ${ }^{1 *}$ \\ 1 Department of Gastric Surgery, State Key Laboratory of Oncology in South China, Collaborative Innovation Center for \\ Cancer Medicine, Sun Yat-sen University Cancer Center, Guangzhou, China, ${ }^{2}$ School of Public Health (Shenzhen), Sun Yat- \\ Sen University, Guangzhou, China, ${ }^{3}$ Department of Gastrointestinal Surgery, The Second Affiliated Hospital of Guangzhou \\ Medical University, Guangzhou, China
}

Background: Despite the implementation of the 8th American Joint Committee on Cancer (AJCC) TNM staging system for gastric cancer (GC) in 2017, it still holds a significant level of stage migration which affects patients' proper classification and accurate prognosis. Here, to reduce this effect, we evaluated the prognostic value of a lymph node ratio (LNR) and established a novel tumor-ratio-metastasis (TRM) staging system.

Method: The data of 15,206 GC patients from the Sun Yat-sen University Cancer Center (Training set; $n=2,032$ ) and the US Surveillance, Epidemiology, and End Results (SEER) database (Validation set; $n=13,174$ ) were analyzed. The training set was classified into 5 LNR categories, based on which the novel TRM staging system was constructed. The overall survival (OS) between the TRM and AJCC TNM systems was compared in the training set and validated in the validation set. The likelihood ratio $x^{2}$, liner trend $x^{2}$, $\mathrm{C}$-index, and Akaike information criterion (AIC) values were used to measure the discriminatory ability between the two different staging systems. Decision curve analyses (DCAs) were conducted to test the clinical value of the two staging systems.

Result: The patients were classified into the following categories: LNR0: 0\%, LNR1: 0\% $<L N R \leq 10 \%$, LNR2: $10 \%<L N R \leq 25 \%$, LNR 3a: $25 \%<L N R \leq 60 \%$, and LNR 3b: $\mathrm{LNR}>60 \%$. Univariate analyses demonstrated that the log-rank $x^{2}$ of the LNR stage (Training/Validation set: $x^{2}=463.1 / 2880.8$ ) was larger than the AJCC pN stage (Training/ Validation set: $\left.x^{2}=281.5 / 2240.8\right)$. For both the training set and validation set, stratified analyses using the Kaplan-Meier method identified significantly heterogeneous OS in every $\mathrm{pN}$ category but only one using the LNR. The TRM staging system had higher likelihood ratio $x^{2}$, liner trend $x^{2}, C$-index and smaller AIC values than the TNM system.

Conclusion: The TRM staging system demonstrated improved homogeneity and discriminatory ability in predicting the prognosis of GC patients compared with the AJCC TNM staging system.

Keywords: lymph node ratio, gastric cancer, staging, prognosis, TNM (8th edition) 


\section{INTRODUCTION}

Gastric Cancer (GC) is still the fifth most frequently diagnosed cancer and the third leading cause of cancer deaths worldwide despite declining incidence in the past years (1-3). The overall trend of GC incidence, which is estimated by age-standardizing among the reference population, may cover up the significant age-specific features. Several studies have indicated that reverse trends of incidence were observed among young people in both the West and the East (4-8), leading to greater loss in lifeexpectancy among younger patients.

Since treatments are directed based on the stage of patients at the time of diagnosis and post-surgery, patients should be accurately staged for optimal treatment. Currently, the most commonly used staging system for GC is the American Joint Committee on Cancer (AJCC) TNM system, which stages patients according to the depth of tumor infiltration (T), number of regional metastatic lymph nodes $(\mathrm{N})$, and status of distant metastasis (M). In the 8th AJCC TNM system, patients with no metastasized LNs, 1-2, 3-6, 7-15 or >15 metastatic LNs are classified as N0, N1, N2, N3a or N3b, respectively. For proper staging, the AJCC recommends at least 16 regional lymph nodes be pathologically assessed. However, the number of resected lymph nodes varies widely, depending on the actual number of regional LNs, surgical technique, surgeons' skills, and/or pathological procedures. This may lead to stage migration, also called the Will Rogers phenomenon $(9,10)$; i.e. if the number of retrieved LNs is less than 16, this could lead to down-migration of the $\mathrm{N}$ stage.

Lymph node ratio (LNR), also called the node ratio (Nr) or metastatic lymph node ratio (MLNR), is defined as the number of MLN divided by the number of retrieved lymph nodes (11). It has been shown as an effective alternative to decrease the risk of stage migration. Several previous studies have analyzed the prognostic role of LNR and found that LNR is an independent prognostic factor for GC. These studies demonstrated that LNR could better stratify the survival of GC patients than the AJCC pN status (12-18). However, such studies were performed mostly using the 7th or earlier edition of the AJCC TNM system. Since the latest AJCC, the 8th TNM staging system has been released and is superior to 7 th edition (19-21). Here, the present study aims to evaluate whether a better $\mathrm{N}$ staging system can be provided by replacing the absolute number of regional node metastasis with lymph node ratio, and whether a novel tumorratio-metastasis (TRM) staging system shows a better performance compared with the 8th edition AJCC TNM system.

\section{METHODS}

\section{Chinese Cohort and Follow-up}

This was a retrospective study comprising of the clinicopathological data of 2,032 GC patients who underwent surgical treatment from January 2000 to June 2017 at the Sun Yat-sen University Cancer Center (SYSUCC; Guangzhou, China). The main eligibility criteria for the study inclusion were histologically confirmed R0 resection. Exclusion criteria were (i) presence of other simultaneous cancer(s) and distant metastasis, (ii) underwent R1 or R2 resection, (iii) had stump or recurrent cancer, (iv) underwent preoperative anti-cancer treatment, (v) died during the perioperative period, and (vi) had incomplete follow-up data. Gastrectomy with lymphadenectomy (D1/D2) was performed by surgeons abiding to the JGCA guidelines, with more than 10 years of experience in performing gastrectomy (22). The data of patients from SYSUCC were used as the training cohort for establishing a hypothetical LNR stage and TRM staging system.

Follow-up assessments including clinical and laboratory examinations were conducted every 3 months for the first 2 years, every 6 months during the 3rd to 5th years, and every 12 months thereafter until death. The primary endpoint was 5-year overall survival (OS). OS was estimated from the date of operation until death or last follow-up contact (February 29, 2020), which was used as a measure to indirectly reflect the prognosis of patients.

\section{Ethical Approval Statement}

This research was approved by the Ethics Committee of the Sun Yat-sen University Cancer Center, and informed consent was granted a waiver due to the retrospective nature of the study.

\section{Western Cohort}

The data of GC patients from the US Surveillance, Epidemiology, and End Results (SEER) database, treated during the year 20042017, was retrieved using the SEER ${ }^{\star}$ Stat software (version 8.3.6). The database named "Incidence - SEER Research Data, 13 Registries, Nov 2019 Sub (1992-2017)", was released in April 2019. Patients who had a "Site and Morphology. CS Schema AJCC 6th Edition" data field of "Stomach" were selected using similar inclusion and exclusion criteria as the Chinese cohort. This Western dataset was used as the validation cohort to confirm the significance of the LNR stage and TRM staging system.

\section{The LNR Stage and TRM Staging System}

The optimal LNR cutoff points were determined using the X-tile software (23) for classifying the LNR into five categories, namely LNR0, LNR1, LNR2, LNR3a, and LNR3b, to maintain consistency with the 8th AJCC nodal categories number (Table 1); based on which the novel TRM staging system was constructed. For the TRM staging system, the same T and M definitions as the 8th AJCC TNM staging system was used, while the $\mathrm{R}$ referred to the LNR categories.

TABLE 1 | TRM/TNM staging system.

\begin{tabular}{lccccc}
\hline & N0/LNR & N1/LNR1 & N2/LNR2 & N3a/LNR3a & N3b/LNR3b \\
\hline T1 & IA & IB & NA & IIB & IIB \\
T2 & IB & IA & IIB & IIA & IIB \\
T3 & II & IIB & IIA & IIB & IIC \\
T4a & IIB & IIA & IIA & IIB & IIC \\
T4b & IIA & IIB & IIB & IIC & IIC
\end{tabular}




\section{Statistical Analysis}

The Kaplan-Meier (KM) method was used to analyze patients' OS. To assess the prognostic performance of the 8th AJCC TNM system, each TNM stage was stratified by TRM stages. In the same way, each TRM stage was stratified by TNM stages to assess the performance of the TRM staging system. The homogeneity across subgroups within each stage was compared using the log-rank test. Moreover, we used the likelihood ratio $x^{2}$ test within the Cox proportional hazard regression model to compare and evaluate the homogeneity of the TRM system and the TNM system. The Akaike information criterion (AIC) value and C-index value, related to a Cox regression model, were calculated for the two systems to measure discriminatory ability. A smaller AIC value and larger C-index value indicate a more desirable model for predicting outcome (24-27). Decision curve analyses, based on the Cox proportional-hazards model, were then applied to both the training set and validation set to compare performance of the TRM staging system and TNM staging system. Twosided $\mathrm{P}$ values were calculated for all tests and a $\mathrm{P}$ value of less than 0.05 was considered statistically significant. Analyses were performed using the SPSS software (version 25.0) and R Studio (version 1.3.1093).

\section{RESULTS}

\section{Patients Characteristics}

The data of 2,032 and 13,174 patients were retrieved from the SYSUCC and SEER databases, respectively. There were 697 female patients and 1,335 male patients in the training set, and 4,894 female patients and 8,280 male patients in the validation set, with a mean age of $57.4 \pm 11.8$ years (range $20-90$ years) and $64.9 \pm 12.8$ years (range $20-85$ years), respectively. The 5-year survival rates of the patients in training and validation set were $64.6 \%$ and $44.3 \%$. The patients clinicopathological characteristics of both groups were listed in Table 2 .

\section{Comparison Between the 8th AJCC pN Stage and LNR Stage}

For the training set, the 5-year OS for the $\mathrm{pN}$ stage categories (N0$\mathrm{N} 3 \mathrm{~b})$ was $86.5 \%, 67.0 \%, 62.9 \%, 45.7 \%$, and $34.7 \%(\mathrm{P}<0.001$, logrank test $x^{2}=281.5$, overall comparisons), respectively. Additionally, pairwise comparison showed significant difference $(\mathrm{P}<0.001)$ between-categories ( $\mathrm{pN} 0$ vs $\mathrm{pN} 1$; $\mathrm{pN} 2$ vs $\mathrm{pN} 3 \mathrm{a}$; $\mathrm{pN} 3 \mathrm{a}$ vs $\mathrm{pN} 3 \mathrm{~b})$, except for $\mathrm{pN} 1$ vs $\mathrm{pN} 2(\mathrm{P}=0.546)$ (Figure 1A).

For the LNR categories (LNR0-LNR3b), the 5-year OS was $86.5 \%, 74.6 \%, 62.6 \%, 45.1 \%$, and $21.3 \%$ ( $\mathrm{P}<0.001$, log-rank test $x^{2}=463.1$, overall comparisons), respectively. Similar pairwise comparison showed significant difference $(\mathrm{P}<0.05)$ within each of the categories (Figure 1B). Then, the $\mathrm{pN}$ with LNR categories were compared. First, each pN category $(\mathrm{N} 1-\mathrm{N} 3 \mathrm{~b})$ was stratified into LNR subgroups. Using the Kaplan-Meier method (log-rank test), significant heterogeneity in 5-year OS was found in each pN category $(4 / 4,100 \%)$. Second, each LNR category (LNR1-
TABLE 2 | Clinic-pathological Factors of training set and validation set.

\begin{tabular}{|c|c|c|c|c|}
\hline \multirow[t]{2}{*}{ Clinic-pathological Factors } & \multicolumn{2}{|c|}{ Training set } & \multicolumn{2}{|c|}{ Validation set } \\
\hline & $n$ & $\%$ & $n$ & $\%$ \\
\hline All & 2032 & & 13174 & \\
\hline \multicolumn{5}{|l|}{ Age } \\
\hline$\leq 39$ & 183 & 9.0 & 363 & 2.8 \\
\hline $40-59$ & 903 & 44.4 & 3244 & 24.6 \\
\hline$\geq 60$ & 946 & 46.6 & 9567 & 72.6 \\
\hline \multicolumn{5}{|l|}{ Gender } \\
\hline Male & 1335 & 65.7 & 8280 & 52.9 \\
\hline Female & 697 & 34.3 & 4894 & 37.1 \\
\hline Tumor size & & & & NA \\
\hline$\leq 4 \mathrm{~cm}$ & 1009 & 49.7 & & \\
\hline$>4 \mathrm{~cm}$ & 987 & 48.6 & & \\
\hline Unknown & 36 & 1.8 & & \\
\hline Histological grade & & & & NA \\
\hline Well & 71 & 3.5 & & \\
\hline Moderately & 240 & 11.8 & & \\
\hline Poorly/Undifferentiated & 1652 & 81.3 & & \\
\hline Unknown & 69 & 3.4 & & \\
\hline Gastrectomy Type & & & & NA \\
\hline Distal & 1122 & 55.2 & & \\
\hline Proximal & 379 & 18.7 & & \\
\hline Total & 449 & 22.1 & & \\
\hline Unknown & 84 & 4.0 & & \\
\hline \multicolumn{5}{|l|}{ No. of LNs } \\
\hline$\leq 15$ & 330 & 16.2 & 6486 & 49.2 \\
\hline$>15$ & 1702 & 83.8 & 6688 & 50.8 \\
\hline \multicolumn{5}{|l|}{ pT stage } \\
\hline $\mathrm{T} 1$ & 382 & 18.8 & 3394 & 25.8 \\
\hline T2 & 234 & 11.5 & 1770 & 13.4 \\
\hline T3 & 798 & 39.3 & 4357 & 33.1 \\
\hline $\mathrm{T} 4 \mathrm{a}$ & 539 & 26.5 & 2801 & 21.3 \\
\hline $\mathrm{T} 4 \mathrm{~b}$ & 79 & 3.9 & 852 & 6.5 \\
\hline \multicolumn{5}{|l|}{ pN stage } \\
\hline NO & 643 & 31.6 & 6067 & 46.1 \\
\hline N1 & 318 & 15.6 & 2341 & 17.8 \\
\hline N2 & 356 & 17.5 & 2032 & 15.4 \\
\hline N3a & 419 & 20.6 & 1779 & 13.5 \\
\hline N3b & 296 & 14.6 & 955 & 7.2 \\
\hline \multicolumn{5}{|l|}{ LNR stage } \\
\hline LNRO & 643 & 31.6 & 6067 & 46.1 \\
\hline LNR1 & 348 & 17.1 & 1348 & 10.2 \\
\hline LNR2 & 360 & 17.7 & 1673 & 12.7 \\
\hline LNR3a & 480 & 23.6 & 2146 & 16.3 \\
\hline LNR3b & 201 & 9.9 & 1940 & 14.7 \\
\hline
\end{tabular}

NA, Not Available.

LNR3b) was also stratified into $\mathrm{pN}$ subgroups. Only significant heterogeneity in the 5 -year OS of the LNR1 category was found $(1 / 4,25 \%)$ (Table 3$)$.

For the validation set, 5 -year OS for the $\mathrm{pN}$ stage categories (N0$\mathrm{N} 3 \mathrm{~b})$ was $62.8 \%, 39.7 \%, 31.6 \%, 20.2 \%$, and $11.7 \%(\mathrm{P}<0.001$, logrank test $x^{2}=2240.8$, overall comparisons), respectively. For the LNR categories (LNR0-LNR3b), the 5-year OS was 62.8\%,50.8\%, $36.4 \%, 24.8 \%$, and $11.9 \%\left(\mathrm{P}<0.001\right.$, log-rank test $x^{2}=2880.8$, overall comparisons), respectively. Pairwise comparison showed that both the $\mathrm{pN}$ and the LNR category had a good discriminatory ability among each category (Figures 2A, B). Then after stratified analyses, significantly heterogeneous 5 -year OS was observed in each of the pN category $(4 / 4,100 \%)$, howreve, significantly heterogeneous 5year OS was only found in LNR2 category $(1 / 4,25 \%)$ (Table 3 ). 
A
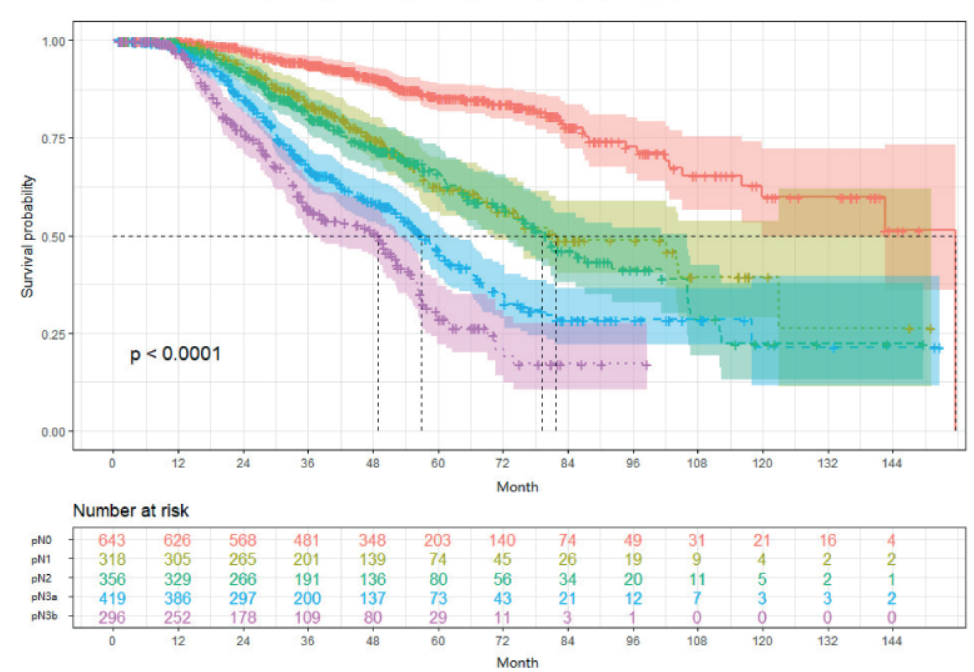

C
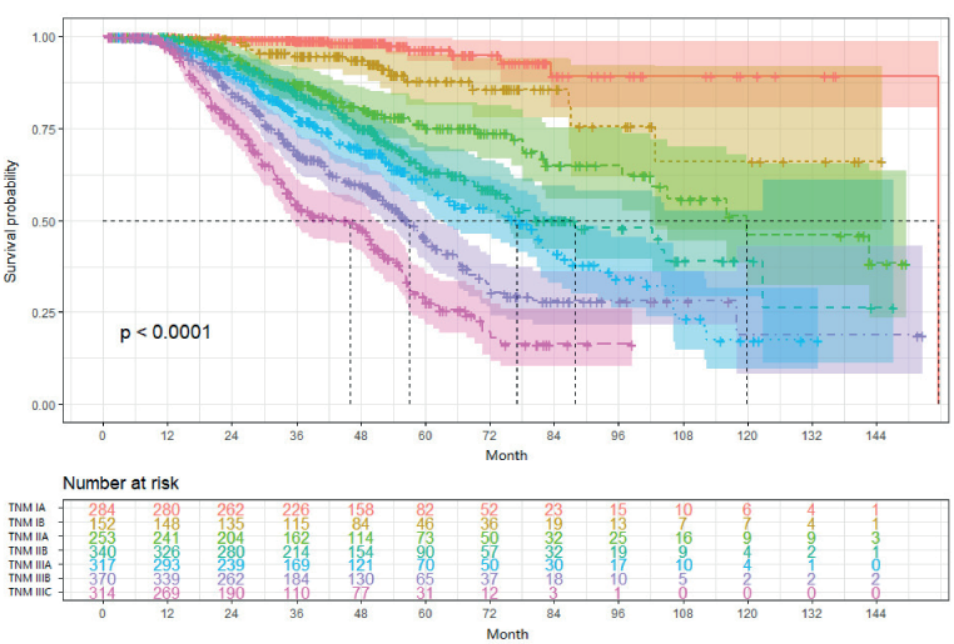

B
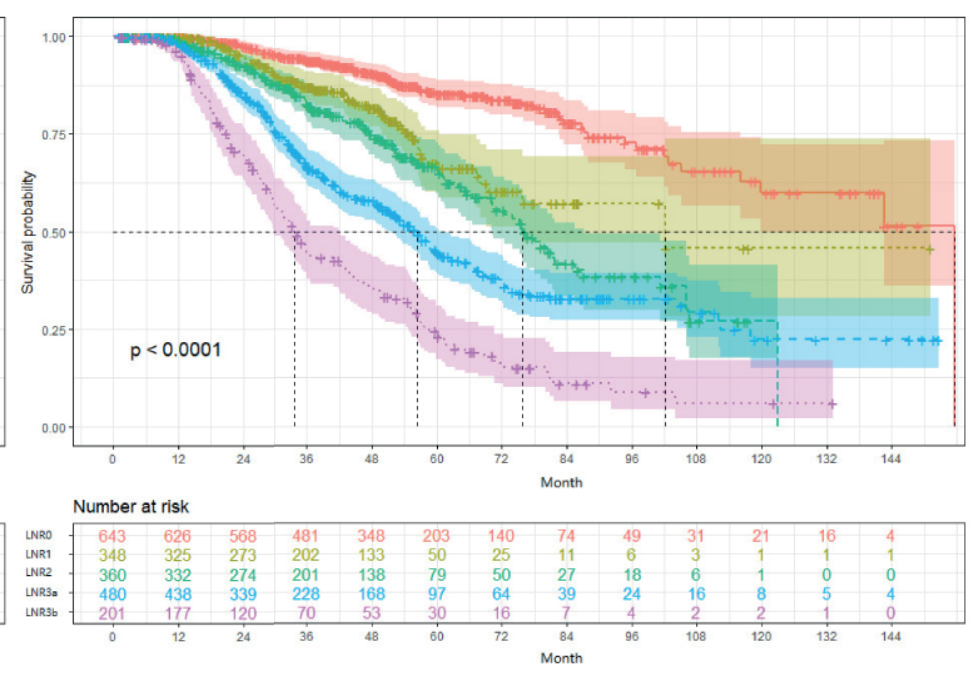

D

TRM Stage $\quad$ TRM IA + TRM IIA + TRM IIA + TRM IIIC

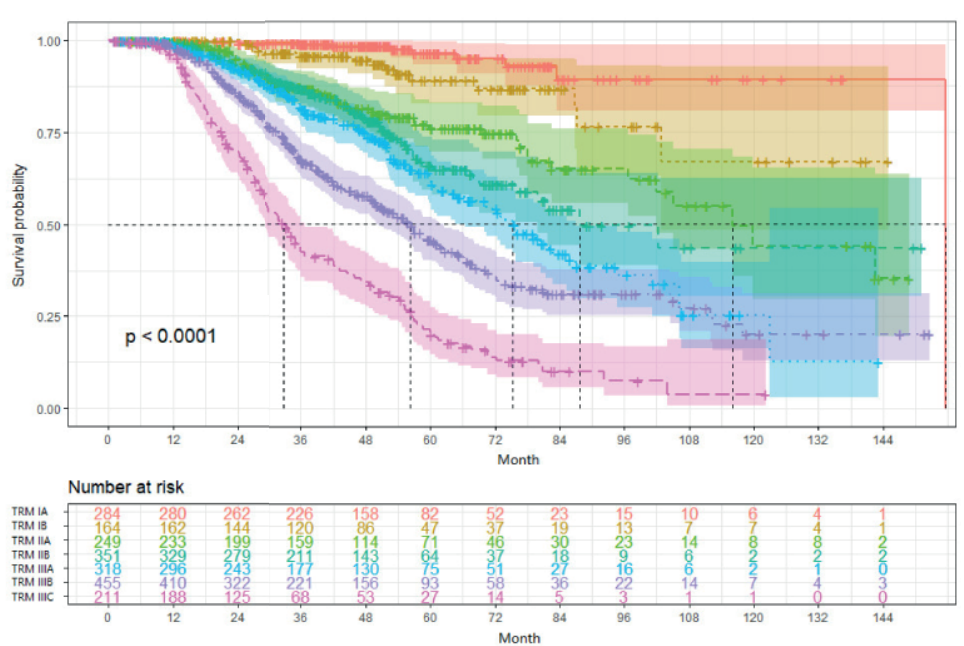

FIGURE 1 | Impact of the $\mathrm{pN}$ (A), LNR (B), TNM (C), and TRM (D) staging on OS in training set. (A) pN stage: pN0 vs pN1, $p<0.001 ; \mathrm{pN1}$ vs pN2, $p=0.546 ; \mathrm{pN} 2$ vs pN3a, $p<0.001 ; \mathrm{pN} 3 a$ vs pN3b, $p<0.001$; (B) LNR stage: LNRO vs LNR1, $p<0.001$; LNR1 vs LNR2, $p=0.010$; LNR2 vs LNR3a, $p<0.001$; LNR3a vs LNR3b, $p<0.001$; (C) TNM staging system: IA vs IB, P = 0.006; IB vs IIA, $p=0.004$; IIA vs IIB, $p=0.016$; IIB vs IIIA, $p=0.101$; IIIA vs IIIB, $p=0.001$; IIIB vs IIIC, $p<0.001$; (D) TRM staging system: IA vs IB, $p=0.020 ;$ IB vs IIA, $p=0.001 ;$ IIA vs IIB, $p=0.185 ; \| B$ vs IIIA, $p=0.024$; IIIA vs IIIB, $p<0.001 ;$ IIIB vs IIIC,

$p<0.001$. LNR, lymph node ratio; TNM, tumor-node-metastasis; TRM, tumor-ratio-metastasis. 
TABLE 3 | Five-Year OS by N Stage and LNR Stage for training set and validation set.

\begin{tabular}{|c|c|c|c|c|c|c|}
\hline \multirow{2}{*}{$\begin{array}{l}\text { Training set } \\
\text { pN stage }\end{array}$} & \multicolumn{5}{|c|}{ LNR stage } & \multirow[t]{2}{*}{$p$} \\
\hline & LNRO & LNR1 & LNR2 & LNR3a & LNR3b & \\
\hline NO & $86.5(643)^{\star}$ & & & & & NA \\
\hline N1 & & $68.7(246)$ & $68.3(54)$ & $38.5(13)$ & $20.0(5)$ & $<0.001$ \\
\hline N2 & & $92.1(98)$ & 60.2 (169) & $43.9(76)$ & $35.2(13)$ & $<0.001$ \\
\hline N3a & & NA (4) & $60.7(130)$ & $43.4(226)$ & $21.6(59)$ & $<0.001$ \\
\hline N3b & & & $51.4(7)$ & $45.6(165)$ & 19.7 (124) & $<0.001$ \\
\hline$P$ & NA & 0.004 & 0.422 & 0.408 & 0.408 & \\
\hline \multirow{2}{*}{$\begin{array}{l}\text { Validation set } \\
\text { pN stage }\end{array}$} & \multicolumn{5}{|c|}{ LNR stage } & $p$ \\
\hline & LNRO & LNR1 & LNR2 & LNR3a & LNR3b & \\
\hline NO & 62.8 (6067) & & & & & NA \\
\hline N1 & & $51.4(1185)$ & $33.0(740)$ & $24.0(231)$ & $6.3(185)$ & $<0.001$ \\
\hline N2 & & $47.4(155)$ & 39.0 (773) & $26.5(850)$ & 17.5 (254) & $<0.001$ \\
\hline N3a & & $19.4(8)$ & 40.5 (155) & 23.7 (905) & $11.4(711)$ & $<0.001$ \\
\hline N3b & & & $0(5)$ & $22.3(160)$ & $9.4(790)$ & $<0.001$ \\
\hline$P$ & NA & 0.262 & 0.028 & 0.864 & 0.061 & \\
\hline
\end{tabular}

*5-year OS rate (\%); (number of patients); NA, Not Available.

$p<0.05$ indicates significant heterogeneity between subgroups.

\section{Comparison Between the 8th AJCC TNM System and Hypothetical TRM System}

First, since the TRM IA stage is equal to TNM IA stage, each TRM stage (except IA) was stratified into TNM stage subgroups to estimate the performance of the novel TRM system. Second, the homogeneity of the 5-year OS for subgroups within every TRM stage was evaluated using the log-rank test. Finally, a same stratified analysis method was performed in each TNM stage (except IA). For the training set, we found that only 2 (IIA, IIB) of the 6 TRM subgroups showed statistically heterogeneous 5year OS, while up to 5 of 6 TNM stage subgroups (except IIA) had statistically heterogeneous 5-year OS (Table 4). The K-M plots of TNM stage and TRM stage were shown in Figures 1C, D. In the validation set, we used the same stratified analyses between the two systems. There was only 1 of 6 TRM stage subgroups (IIIC) showed statistically heterogeneous 5-year OS, while all of the 6 TNM stage subgroups showed statistically heterogeneous (Table 4). The K-M plots of TNM stage and TRM stage were shown in Figures 2C, D.

\section{Comparison Between the Two Systems Within the Patients With Over 15 LNs Retrieved in Validation Set}

For patients with more than 15 examined lymph nodes, the TRM staging system was also superior to TNM staging system. The log-rank $x^{2}$ of LNR stage $\left(x^{2}=1723.7\right)$ was larger than that of $\mathrm{pN}$ stage $\left(x^{2}=1552.3\right)$. For the stratified analyses, every $\mathrm{pN}$ stage (except $\mathrm{pN} 0$ ) showed significantly heterogeneous 5year OS (pN1: $\mathrm{P}=0.004 ; \mathrm{pN} 2-\mathrm{pN} 3 \mathrm{~b}$ : $\mathrm{P}<0.001$ ) by stratifying into LNR subgroups, but only LNR3b showed significantly heterogeneous results(LNR3b: $\mathrm{P}=0.018$; LNR1: $\mathrm{P}=0.064$; LNR2: $\mathrm{P}=0.953$; LNR3a: $\mathrm{P}=0.654$ ). Additionally, 5 of $6 \mathrm{TNM}$ stages (except IIB) showed significantly heterogeneous by stratified into TRM subgroups (TNM IB: $\mathrm{P}=0.014$; TNM IIA: $\mathrm{P}=0.009$; TNM IIB: $\mathrm{P}=0.092$; TNM IIAB: $\mathrm{P}=0.001$; TNM IIIB/IIIC: $\mathrm{P}<0.001$ ), while only TRM IIIC showed significantly heterogeneous results
(TRM IB: $\mathrm{P}=0.065$; TRM IIA: $\mathrm{P}=0.513$; TRM IIB: $\mathrm{P}=0.194$; TRM IIAB: $\mathrm{P}=0.706$; TNM IIIB: $\mathrm{P}=0.749$; TRM IIIC: $\mathrm{P}<0.001$ ). Similar results were observed for the patients with over 15 LNs retrieved in the training set. Due to space limitations, there is no further description here.

\section{Compared the Performance Between the TRM and TNM Systems}

In this part, the performance between the TRM staging system and TNM staging system, including the comparison between LNR and $\mathrm{pN}$ stage, was evaluated by the likelihood ratio $x^{2}$, linear trend $x^{2}, \mathrm{C}$-index, and AIC value (Table 5). For both the training set and validation set, the TRM staging system showed better homogeneity (larger likelihood ratio $x^{2}$ ), discriminatory ability, and monotonicity of gradients (larger linear trend $x^{2}$ ) than the TNM staging system. Moreover, the C-index of the TRM staging system was larger than that of the TNM staging system, while the AIC value of the TRM staging system was smaller than the TNM staging system. The results of the C-index and AIC value also indicate that the performance of the TRM system is better than the TNM system. The results of decision curve analyses comparing the performance between the TRM and TNM systems are shown in Figure 3, which indicate that both the LNR stage and TRM stage have greater net benefit than the $\mathrm{pN}$ stage and TNM stage, both in the training set and validation set.

\section{DISCUSSION}

In the present study, we constructed a novel LNR stage as an alternative to $\mathrm{pN}$ stage, and replaced $\mathrm{pN}$ of the TNM staging system with LNR so as to set up the TRM staging system. Our results indicate that both the LNR stage and TRM staging system could bring more accurate patients stratification and more prognostic value compared with the $\mathrm{pN}$ stage and TNM staging system (AJCC 8th edition). 
A

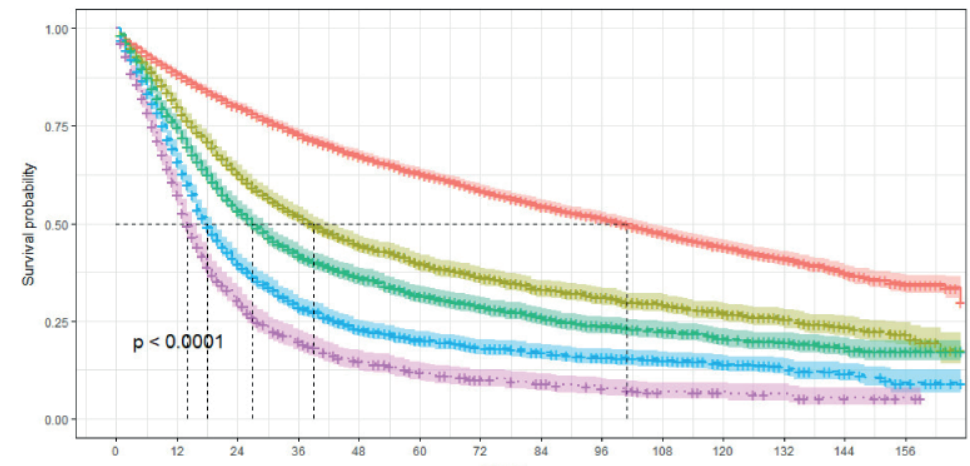

\section{Number at risk}

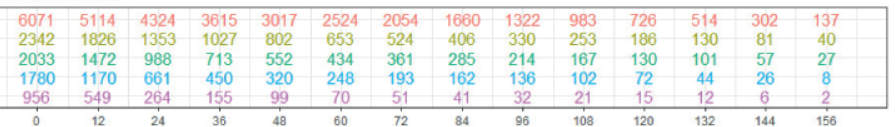

C

TNM stage + TNM IA + TNM IIA + TNM IIIA + TNM IIIC
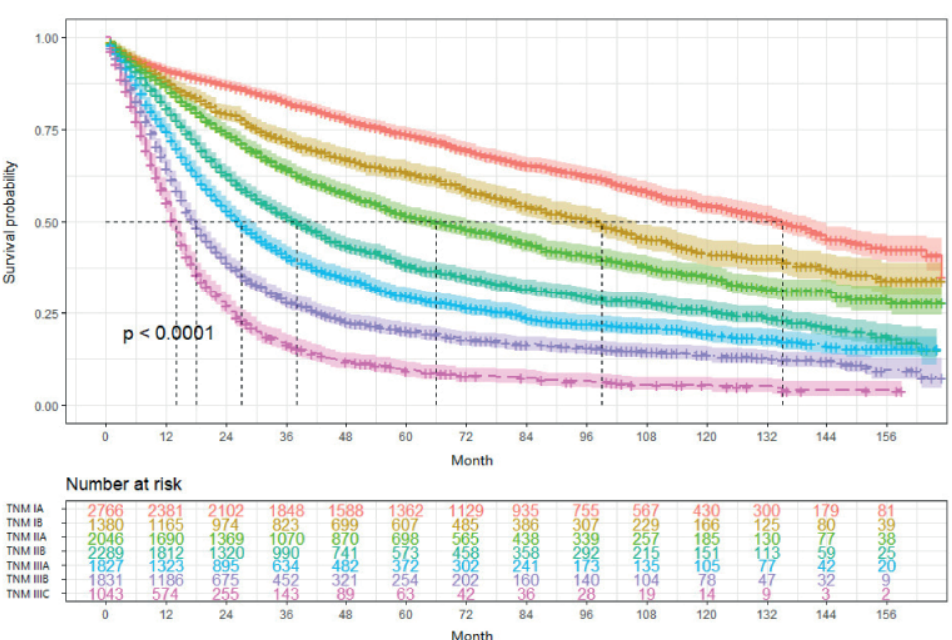

LNR stage $=$ LNR0 $F$-LNR1 + LNR2 + LNR3a + LNR3b
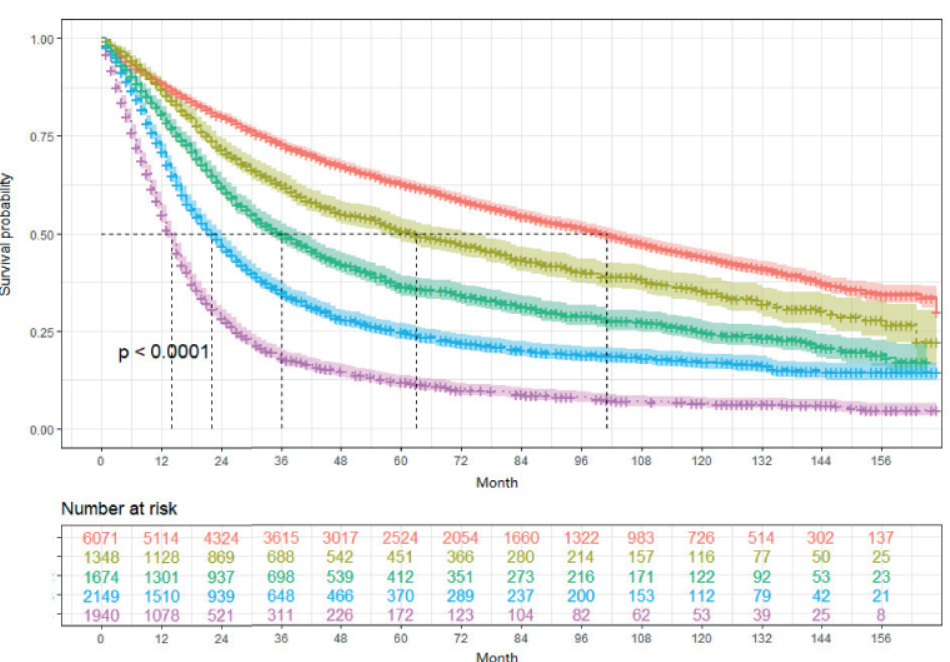

TRM stage $\quad+$ TRM IA + TRM IIA + TRM IIIA + TRM IIIC

D
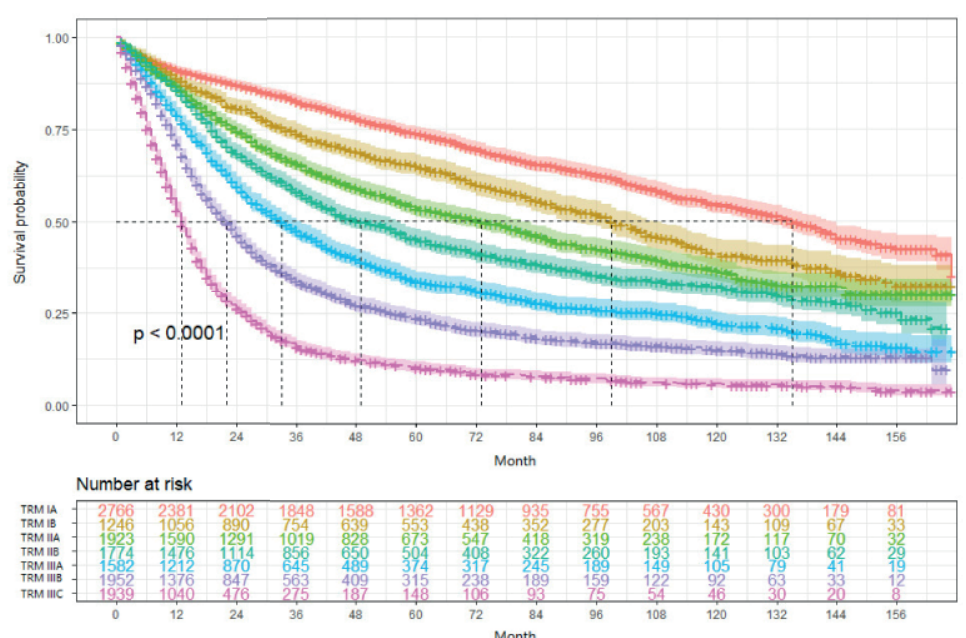

FIGURE 2 Impact of the pN (A), LNR (B), TNM (C), and TRM (D) staging on OS in validation set. (A) pN stage: pNO vs pN1, $p<0.001 ; \mathrm{pN} 1$ vs pN2, $p<0.001 ; \mathrm{pN} 2$ vs pN3a, $p<0.001 ; \mathrm{pN} 3 a$ vs pN3b, $p<0.001$; (B) LNR stage: LNR0 vs LNR1, $p<0.001$; LNR1 vs LNR2, $p<0.001$; LNR2 vs LNR3a, $p<0.001$; LNR3a vs LNR3b, $p<0.001$; (C) TNM staging system: IA vs IB, $p<0.001 ;$ IB vs IIA, $p<0.001$; IIA vs IIB, $p<0.001$; IIB vs IIIA, $p<0.001$; IIIA vs IIIB, $p<0.001$; IIIB vs IIIC, $p<0.001$; (D) TRM staging system: IA vs IB, $p<0.001$; IB vs IIA, $p<0.001 ; \|$ AA vs IIB, $p<0.001$; IIB vs IIIA, $p<0.001$; IIIA vs IIIB, $p<0.001$; IIIB vs IIIC, $p<0.001$. LNR, lymph node ratio; TNM, tumor-node-metastasis; TRM, tumor-ratio-metastasis. 
TABLE 4 | Five-Year OS by TNM Stage and TRM Stage for training set and validation set.

\begin{tabular}{|c|c|c|c|c|c|c|c|c|}
\hline \multirow{2}{*}{$\begin{array}{l}\text { Training set } \\
\text { TNM }\end{array}$} & \multicolumn{7}{|c|}{ TRM } & \multirow[t]{2}{*}{$p$} \\
\hline & IA & IB & IIA & IIB & IIIA & IIIB & IIIC & \\
\hline IA & $96.7(284)^{\star}$ & & & & & & & NA \\
\hline IB & & $91.1(150)$ & $50.0(2)$ & & & & & $<0.001$ \\
\hline$\| \mathrm{A}$ & & NA (13) & 76.3 (227) & $73.8(13)$ & & & & 0.360 \\
\hline$\| B$ & & NA (1) & NA (20) & 65.3 (253) & $71.4(47)$ & $40.0(15)$ & $25.0(4)$ & $<0.001$ \\
\hline IIIA & & & & $89.2(77)$ & $54.8(155)$ & $41.6(74)$ & $31.8(11)$ & $<0.001$ \\
\hline IIIB & & & & NA (6) & $57.2(109)$ & 42.3 (208) & $18.0(47)$ & $<0.001$ \\
\hline IIIC & & & & & $51.4(7)$ & $45.2(158)$ & $19.0(149)$ & $<0.001$ \\
\hline$P$ & NA & 0.661 & $<0.001$ & 0.020 & 0.323 & 0.470 & 0.608 & \\
\hline Validation set & \multicolumn{7}{|c|}{ TRM } & $p$ \\
\hline TNM & IA & IB & IIA & IIB & IIIA & IIIB & IIIC & \\
\hline IA & 73.8 (2766) & & & & & & & NA \\
\hline IB & & $65.2(1231)$ & $50.0(110)$ & $60.0(25)$ & 35.7 (14) & & & $<0.001$ \\
\hline$\| \mathrm{A}$ & & $50.9(15)$ & 53.7 (1782) & $41.3(184)$ & $25.0(45)$ & $45.9(20)$ & & $<0.001$ \\
\hline IIB & & & $53.1(30)$ & $45.3(1447)$ & $30.3(538)$ & $20.4(165)$ & $12.2(109)$ & $<0.001$ \\
\hline IIIA & & & NA (1) & $47.6(112)$ & $35.0(843)$ & 23.5 (679) & $17.7(192)$ & $<0.001$ \\
\hline IIIB & & & & $22.2(6)$ & $40.4(137)$ & 24.1 (943) & $11.5(745)$ & $<0.001$ \\
\hline $\mathrm{IIIC}$ & & & & & $40.0(5)$ & $22.8(145)$ & 7.1 (893) & $<0.001$ \\
\hline$P$ & NA & 0.215 & 0.519 & 0.255 & 0.230 & 0.185 & 0.005 & \\
\hline
\end{tabular}

*5-year OS rate (\%);(number of patients); NA, Not Available.

TABLE 5 | Comparison of the performance of the AJCC TNM staging system and TRM staging system.

\begin{tabular}{|c|c|c|c|c|c|c|}
\hline Group & Classification & Subgroups & Likelihood ratio $x^{2}$ & Linear trend $x^{2}$ & C-index & AIC value \\
\hline \multirow[t]{4}{*}{ Training set } & pN stage & NO-N3b & 281.41 & 185.39 & 0.69 & 8219.14 \\
\hline & LNR stage & LNRO-LNR3b & 462.92 & 291.99 & 0.72 & 8156.79 \\
\hline & TNM stage & $|A-||| C$ & 340.42 & 248.16 & 0.71 & 8130.17 \\
\hline & TRM stage & $|A-||| C$ & 534.39 & 332.32 & 0.73 & 8075.77 \\
\hline \multirow[t]{4}{*}{ Validation set (Total) } & pN stage & NO-N3b & 2202.81 & 1579.91 & 0.65 & 132895.23 \\
\hline & LNR stage & LNRO-LNR3b & 2831.63 & 1946.94 & 0.66 & 132447.64 \\
\hline & TNM stage & $|A-||| C$ & 2768.89 & 2100.87 & 0.67 & 132301.03 \\
\hline & TRM stage & $|A-||| C$ & 3353.71 & 2347.63 & 0.68 & 131988.44 \\
\hline \multirow[t]{4}{*}{ Validation set (> 15 LNs) } & pN stage & NO-N3b & 1526.89 & 1100.26 & 0.68 & 57880.95 \\
\hline & LNR stage & LNRO-LNR3b & 1695.30 & 1158.17 & 0.68 & 57782.85 \\
\hline & TNM stage & $|A-||| C$ & 1762.64 & 1235.62 & 0.69 & 57701.60 \\
\hline & TRM stage & $|A-||| C$ & 1908.22 & 1281.39 & 0.70 & 57624.00 \\
\hline
\end{tabular}

In 2002, metastatic lymph node ratio (MLR) was put forward and used to predict the prognosis of GC patients for the first time (17). After that, several studies have confirmed that staging by the LNR rather than absolute number of MLN could better predict prognosis of gastric cancer patients. For instance, Xiao et al. (28) analyzed the prognosis of 1,042 GC patients who underwent D2 gastrectomy with less than 16 retrieved lymph nodes and divided LNR into four subgroups $(0 \%, 1 \%-30 \%, 31 \%$ $50 \%,>50 \%)$. They demonstrated that using LNR stage could predict the prognosis better than $\mathrm{pN}$ stage when the number of lymph nodes examined was less than 15 . Lee et al (29) analyzed 3284 patients from eight institutions and categorized LNR into five subgroups $(0,0-0.06,0.06-0.27,0.27-0.49$, and $>0.49)$. They demonstrated that LNR stage was a better predictor of prognosis for GC patients than the $\mathrm{pN}$ stage. Wang et al. (13) analyzed 18,043 gastric cancer patients from SEER database and classified LNR into five subgroups $(0,0<\mathrm{LNR} \leq 1 / 15,1 / 15<\mathrm{LNR} \leq 3 / 10$,
$3 / 10<\mathrm{LNR} \leq 7 / 10$ and $\mathrm{LNR}>7 / 10$ ). They found that using the TRM system caused less misclassification than the TNM system ( $12 \%$ vs $57 \%)$.

However, the previous studies had several limitations: (i) The sample size included in the study was relatively small (a few hundred to several thousands) or only from a single institution. (ii) Most previous studies drew their conclusion based on old versions of AJCC TNM edition rather than the new version. (iii) Most previous studies only compared the prognosis value of LNR and pN staging. They did not combine the LNR stage and $\mathrm{pT}$ stage to construct a hypothetical TRM staging system. (iv) Most previous studies determined the cut points of LNR by using the so-called 'best cut-off method by log-rank test', ignoring the fact that LNR should be a continuous variable.

In the present study, we avoided the limitations mentioned above. We analyzed 15,206 GC patients from the Sun Yat-sen University Cancer Center (Training set; $\mathrm{n}=2,032$ ) and the US 

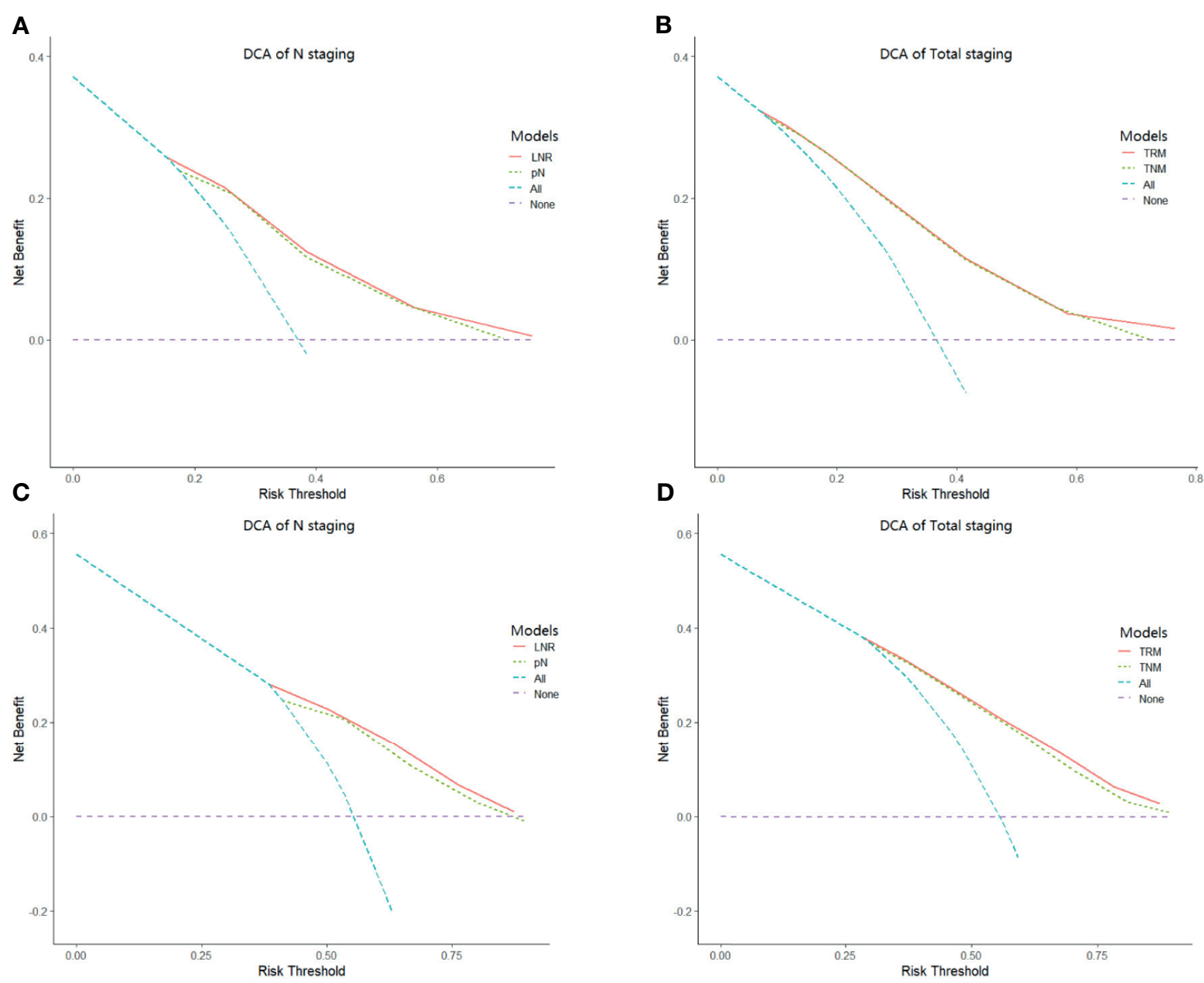

FIGURE 3 | Results of decision curve analysis. Results of decision curve analysis pN vs LNR (A) and TNM vs TRM (B) in the training set; pN vs LNR (C) and TNM vs TRM (D) in the validation set.

Surveillance, Epidemiology, and End Results (SEER) database (Validation set; $\mathrm{n}=13,174$ ). We did not neglect the differences in the multimodal treatment of gastric cancer between East and West (30-32). Furthermore, we fully considered the LNR as a continuous variable, and we used the X-tile software to determine the optimal cut-off point. And we demonstrated that TRM staging system showed the superiority to the 8th AJCC TNM staging system for the reasons as follows: (i) In univariate analysis, the log-rank $x^{2}$ of LNR stage (training set: 463.1; validation set: 2880.8 ) was larger than that of $\mathrm{pN}$ stage (training set: 281.5; validation set: 2240.8 ), which indicated a higher statistical significance. Stratified analyses using the Kaplan-Meier method identified significantly heterogeneous OS in every pN category but only one LNR category (Table 3 ). (ii) In univariate analysis, the log-rank $x^{2}$ of TRM stage (training set: 534.58; validation set: 3412.03 ) was larger than that of TNM stage (training set: 340.54 ; validation set: 2816.64 ), which indicated a higher statistical significance. Stratified analyses identified significantly heterogeneous OS in almost every TNM stage but only one TRM stage (Table 4). (iii) For patients with $>15$ lymph nodes examined in the validation set, stratified analyses showed that five of the six TNM stages (except IIB) showed significantly heterogeneous results, while only TRM IIIC showed significantly heterogeneous results.

Based on Ueno et al (27), regarding the discriminatory ability between different groups, the monotonicity of gradients reflected in the relationship between stages and OS, and the homogeneity within subgroups, a model with larger linear trend $x^{2}$ and the likelihood ratio $x^{2}$ was considered as the better model. In our study, the TRM staging system had better discriminatory ability, monotonicity of gradients (larger liner trend $x^{2}$ ), and homogeneity (larger likelihood ratio $x^{2}$ ). Furthermore, the TRM staging system with a smaller AIC value and a lager $\mathrm{C}$-index value indicated the better prognostic stratification. These results confirmed that the TRM staging system could predict the prognosis of GC patients better than the TNM staging system (Table 5). And moreover, the results of decision curve analyses indicate that the TRM staging system has a greater net 
benefit than the TNM staging system. Although our study demonstrated the superiority of the TRM staging system, it does not mean the surgeons could perform a lesser extent of lymph node dissection. Actually, retrieving as many as possible lymph nodes could predict the prognosis of patients better (33).

There were several limitations in this study. First, as a retrospective study, some data were inevitably missing or not applicable for analysis. For instance, we did not include data on the adjuvant chemotherapy in our study. If postoperative treatments varied greatly between patients, that could bring confounding effects to the study. However, as patients in the training set were from a single cancer center, and physicians would normally prescribe 5-fluorouracil (5-FU) based recipe for those TNM IIA or higher staged GC patients according to latest GC guidelines, the postoperative treatments for our patients were relatively homogeneous. So we did not include this data in the present. Second, our TRM staging system was similar to the TNM staging system. We just replaced the $\mathrm{pN}$ stage with the LNR stage, based on which the novel TRM staging system was constructed. Actually, we could improve our TRM staging system with the better combination of the LNR stage and pT stage, but we think that the staging system will include additional non-anatomical factors in the future.

\section{CONCLUSION}

Our findings showed that the LNR and TRM staging systems could better estimate the 5-year OS than the 8th edition AJCC $\mathrm{pN}$ staging and TNM staging system, due to less stage migration. However, a more extensive lymph node dissection and to standardize the LNR cutoff value is still needed to extend and use LNR/TRM staging system.

\section{REFERENCES}

1. Bray F, Ferlay J, Soerjomataram I, Siegel RL, Torre LA, Jemal A. Global Cancer Statistics 2018: GLOBOCAN Estimates of Incidence and Mortality Worldwide for 36 Cancers in 185 Countries. CA Cancer J Clin (2018) 68 (6):394-424. doi: 10.3322/caac. 21492

2. Venerito M, Link A, Rokkas T, Malfertheiner P. Review: Gastric CancerClinical Aspects. Helicobacter (2019) 24(S1):e12643. doi: 10.1111/hel.12643

3. Gao K, Wu J. National Trend of Gastric Cancer Mortality in China (20032015): A Population-Based Study. Cancer Commun (2019) 39(1):1-5. doi: 10.1186/s40880-019-0372-x

4. Merchant SJ, Kim J, Choi AH, Sun V, Chao J, Nelson R. A Rising Trend in the Incidence of Advanced Gastric Cancer in Young Hispanic Men. Gastric Cancer (2017) 20(2):226-34. doi: 10.1007/s10120-016-0603-7

5. Anderson WF, Rabkin CS, Turner N, Fraumeni JF, Rosenberg PS, Camargo MC. The Changing Face of Noncardia Gastric Cancer Incidence Among US Non-Hispanic White. JNCI: J Natl Cancer Inst (2018) 110(6):608-15. doi: 10.1093/jnci/djx262

6. Takatsu Y, Hiki N, Nunobe S, Ohashi M, Honda M, Yamaguchi T, et al. Clinicopathological Features of Gastric Cancer in Young Patients. Gastric Cancer (2016) 19(2):472-8. doi: 10.1007/s10120-015-0484-1

7. Anderson WF, Camargo MC, Fraumeni JFJr., Correa P, Rosenberg PS, Rabkin CS. Age-Specific Trends in Incidence of Noncardia Gastric Cancer in US Adults. JAMA (2010) 303(17):1723-8. doi: 10.1001/jama.2010.496

\section{DATA AVAILABILITY STATEMENT}

The raw data supporting the conclusions of this article will be made available by the authors, without undue reservation.

\section{ETHICS STATEMENT}

The studies involving human participants were reviewed and approved by Ethics Committee of the Sun Yat-Sen University Cancer Center. Written informed consent for participation was not required for this study in accordance with the national legislation and the institutional requirements.

\section{AUTHOR CONTRIBUTIONS}

All authors took part in conception and design of this work, acquisition and collection of data, analysis of data, and participated in writing and reviewing this manuscript. All authors contributed to the article and approved the submitted version.

\section{FUNDING}

This project was supported by 1 . Chinese Society of Clinical Oncology (Roche foundation grants No: Y-2019Roche-157), 2. Science and Technology Project of Guangdong (grant No. 2014A020212331) and 3. National Natural Science Foundation of China (grant No.82001672).

8. Alston RD, Geraci M, Eden TO, Moran A, Rowan S, Birch JM. Changes in Cancer Incidence in Teenagers and Young Adults (Ages 13 to 24 Years) in England 1979-2003. Cancer (2008) 113(10):2807-15. doi: 10.1002/cncr.23901

9. Kong SH, Lee HJ, Ahn HS, Kim JW, Kim WH, Lee KU, et al. Stage Migration Effect on Survival in Gastric Cancer Surgery With Extended Lymphadenectomy: The Reappraisal of Positive Lymph Node Ratio as a Proper N-Staging. Ann Surg (2012) 255(1):50-8. doi: 10.1097/SLA.0b013e31821d4d75

10. Feinstein AR, Sosin DM, Wells CK. The Will Rogers Phenomenon. Stage Migration and New Diagnostic Techniques as a Source of Misleading Statistics for Survival in Cancer. N Engl J Med (1985) 312(25):1604-8. doi: 10.1056/ NEJM198506203122504

11. Okusa T, Nakane Y, Boku T, Takada H, Yamamura M, Hioki K, et al. Quantitative Analysis of Nodal Involvement With Respect to Survival Rate After Curative Gastrectomy for Carcinoma. Surg Gynecol Obstet (1990) 170(6):488-94.

12. Kutlu OC, Watchell M, Dissanaike S. Metastatic Lymph Node Ratio Successfully Predicts Prognosis in Western Gastric Cancer Patients. Surg Oncol (2015) 24(2):84-8. doi: 10.1016/j.suronc.2015.03.001

13. Wang J, Dang P, Raut CP, Pandalai PK, Maduekwe UN, Rattner DW, et al. Comparison of a Lymph Node Ratio-Based Staging System With the 7th AJCC System for Gastric Cancer: Analysis of 18,043 Patients From the SEER Database. Ann Surg (2012) 255(3):478-85. doi: 10.1097/SLA.0b013e31824857e2

14. Wang W, Xu DZ, Li YF, Guan YX, Sun XW, Chen YB, et al. Tumor-ratiometastasis Staging System as an Alternative to the 7th Edition UICC TNM System in Gastric Cancer After D2 Resection-Results of a Single-Institution 
Study of 1343 Chinese Patients. Ann Oncol (2011) 22(9):2049-56. doi: 10.1093/annonc/mdq716

15. Marchet A, Mocellin S, Ambrosi A, de Manzoni G, Di Leo A, Marrelli D, et al. The Prognostic Value of N-Ratio in Patients With Gastric Cancer: Validation in a Large, Multicenter Series. Eur J Surg Oncol (2008) 34(2):159-65. doi: 10.1016/j.ejso.2007.04.018

16. Celen O, Yildirim E, Berberoglu U. Prognostic Impact of Positive Lymph Node Ratio in Gastric Carcinoma. J Surg Oncol (2007) 96(2):95-101. doi: 10.1002/jso.20797

17. Inoue $\mathrm{K}$, Nakane $\mathrm{Y}$, Iiyama $\mathrm{H}$, Sato $\mathrm{M}$, Kanbara $\mathrm{T}$, Nakai $\mathrm{K}$, et al. The Superiority of Ratio-Based Lymph Node Staging in Gastric Carcinoma. Ann Surg Oncol (2002) 9(1):27-34. doi: 10.1245/aso.2002.9.1.27

18. Hwang JE, Kim H, Shim H-J, Bae W-K, Hwang E-C, Jeong O, et al. Lymph-Node Ratio is an Important Clinical Determinant for Selecting the Appropriate Adjuvant Chemotherapy Regimen for Curative D2-Resected Gastric Cancer. J Cancer Res Clin Oncol (2019) 145(8):2157-66. doi: 10.1007/s00432-019-02963-7

19. Graziosi L, Marino E, Donini A. Survival Comparison in Gastric Cancer Patients Between 7th and 8th Edition of the AJCC TNM Staging System: The First Western Single Center Experience. Eur J Surg Oncol (2019) 45(6):1105-8. doi: 10.1016/j.ejso.2018.12.010

20. Cao L-L, Lu J, Li P, Xie J-W, Wang J-B, Lin J-X, et al. Evaluation of the Eighth Edition of the American Joint Committee on Cancer TNM Staging System for Gastric Cancer: An Analysis of 7371 Patients in the SEER Database. Gastroenterol Res Pract (2019) 2019:6294382. doi: 10.1155/2019/6294382

21. Liu J-Y, Peng C-W, Yang X-J, Huang C-Q, Li Y. The Prognosis Role of AJCC/ UICC 8 Edition Staging System in Gastric Cancer, a Retrospective Analysis. Am J Transl Res (2018) 10(1):292-303.

22. Japanese GCA. Japanese Classification of Gastric Carcinoma - 2nd English Edition. Gastric Cancer (1998) 1(1):10-24. doi: 10.1007/PL00011681

23. Camp RL, Dolled-Filhart M, Rimm DL. X-Tile: A New Bio-Informatics Tool for Biomarker Assessment and Outcome-Based Cut-Point Optimization. Clin Cancer Res (2004) 10(21):7252-9. doi: 10.1158/1078-0432.CCR-04-0713

24. Tez M, Zulfikaroglu B. Comparison of 7 Staging Systems for Patients With Hepatocellular Carcinoma Undergoing Transarterial Chemoembolization. Cancer (2008) 112(12):2820-21. doi: 10.1002/cncr.23506

25. Kee K-M, Wang J-H, Lee C-M, Chen C-L, Changchien C-S, Hu T-H, et al. Validation of Clinical AJCC/UICC TNM Staging System for Hepatocellular Carcinoma: Analysis of 5,613 Cases From a Medical Center in Southern Taiwan. Int J Cancer (2007) 120(12):2650-5. doi: 10.1002/ijc.22616

26. Hsu P-K, Wu Y-C, Chou T-Y, Huang C-S, Hsu W-H. Comparison of the 6th and 7th Editions of the American Joint Committee on Cancer TumorNode-Metastasis Staging System in Patients With Resected Esophageal Carcinoma. Ann Thorac Surg (2010) 89(4):1024-31. doi: 10.1016/ j.athoracsur.2010.01.017
27. Ueno S, Tanabe G, Sako K, Hiwaki T, Hokotate H, Fukukura Y, et al. Discrimination Value of the New Western Prognostic System (CLIP Score) for Hepatocellular Carcinoma in 662 Japanese Patients. Cancer of the Liver Italian Program. Hepatology (2001) 34(3):529-34. doi: 10.1053/ jhep.2001.27219

28. Xiao LB, Yu JX, Wu WH, Xu FF, Yang SB. Superiority of Metastatic Lymph Node Ratio to the 7th Edition UICC N Staging in Gastric Cancer. World J Gastroenterol (2011) 17(46):5123-30. doi: 10.3748/wjg.v17.i46.5123

29. Lee JH, Kang JW, Nam BH, Cho GS, Hyung WJ, Kim MC, et al. Correlation Between Lymph Node Count and Survival and a Reappraisal of Lymph Node Ratio as a Predictor of Survival in Gastric Cancer: A Multi-Institutional Cohort Study. Eur J Surg Oncol (2017) 43(2):432-9. doi: 10.1016/j.ejso. 2016.09.007

30. Russo A, Li P, Strong VE. Differences in the Multimodal Treatment of Gastric Cancer: East Versus West. J Surg Oncol (2017) 115(5):603-14. doi: 10.1002/ jso. 24517

31. Randle RW, Swords DS, Levine EA, Fino NF, Squires MH, Poultsides G, et al. Optimal Extent of Lymphadenectomy for Gastric Adenocarcinoma: A 7 Institution Study of the U.S. Gastric Cancer Collaborative. J Surg Oncol (2016) 113(7):750-5. doi: 10.1002/jso.24227

32. Hartgrink $\mathrm{HH}$, van de Velde $\mathrm{CJH}$, Putter $\mathrm{H}$, Bonenkamp JJ, Klein Kranenbarg E, Songun I, et al. Extended Lymph Node Dissection for Gastric Cancer: Who may Benefit? Final Results of the Randomized Dutch Gastric Cancer Group Trial. J Clin Oncol (2004) 22(11):2069-77. doi: 10.1200/ JCO.2004.08.026

33. Liu Y-Y, Fang W-L, Wang F, Hsu J-T, Tsai C-Y, Liu K-H, et al. Does a Higher Cutoff Value of Lymph Node Retrieval Substantially Improve Survival in Patients With Advanced Gastric Cancer?-Time to Embrace a New Digit. Oncol (2017) 22(1):97-106. doi: 10.1634/theoncologist.2016-0239

Conflict of Interest: The authors declare that the research was conducted in the absence of any commercial or financial relationships that could be construed as a potential conflict of interest.

The RE ZX declared a shared affiliation with the authors to the handling editor at time of review.

Copyright ( 2021 Zhang, Ding, Xu, Ou, Feng, Wang, Wang, Liang, Chen, Zhou and Qiu. This is an open-access article distributed under the terms of the Creative Commons Attribution License (CC BY). The use, distribution or reproduction in other forums is permitted, provided the original author(s) and the copyright owner(s) are credited and that the original publication in this journal is cited, in accordance with accepted academic practice. No use, distribution or reproduction is permitted which does not comply with these terms. 